\title{
Nonperturbative Renormalon Structure of Infrared Unstable Theories
}

\author{
A. Duncan and S. Pernice \\ Department of Physics and Astronomy \\ University of Pittsburgh, Pittsburgh, PA 15620 \\ June 14, 1994 \\ e-mail: tony@dectony.phyast.pitt.edu,sergio@charm.pas.rochester.edu
}




\begin{abstract}
The properties of a generalized version of the Borel Transform in infrared unstable theories with dynamical mass generation are studied. The reconstruction of the nonperturbative structure is unambiguous in this version. Various methods for extracting the singularity structure of the Borel Transform for lattice formulations of such theories are explored, and illustrated explicitly with the $\mathrm{O}(\mathrm{N})$ sigma model. The status of the first infrared renormalon in QCD is discussed. The feasibility of a proposed technique for analytically continuing from the left hand Borel plane (where nonperturbative information is available via simulation of lattice field theory) to the positive real axis is examined using the sigma model.
\end{abstract}




\section{INTRODUCTION}

The problem of divergence of (renormalized) perturbation theory, which can be traced back to the seminal work of Dyson in quantum electrodynamics

[回], is now recognized to lie at the core of any precise analytic understanding of the nonperturbative structure of field theory. The need to face this problem squarely is particularly apparent in massless quantum chromodynamics (QCD), where the underlying dynamics is specified by a single parameter $N$ (for gauge group $S U(N)$ ). Dimensionless ratios of hadron masses in this theory depend only on $N$, which is of order unity $(N=3)$ in the physical world. Apart from the technically intractable $1 / N$ expansion, there is no natural intrinsic expansion parameter for the spectral and other low-energy properties of the theory. If one insists on computing such quantities in a conventional weak-coupling expansion, they either vanish formally to all orders, or yield asymptotic expansions in terms of a running coupling which is naturally of order unity. Such expansions are quantitatively useless in the absence of a reliable "resummation" procedure.

Certain superrenormalizable field-theories (e.g $\phi_{1,2,3}^{4}$ in the unbroken phase) have been shown rigorously [2], [3] to possess Borel-summable perturbative expansions, so that the full content of the theory (at least, informationtheoretically) is exhausted by perturbation theory. The spontaneously broken phases of these theories is typically not Borel-summable, but even here an optimized reorganization of the perturbation theory can be shown rigorously [4 to converge to the exact partition function, provide the theory is formulated at finite volume. The failure of Borel-summability is far more 
severe in QCD [5], and no systematic analytic procedure is known, even in principle, whereby the full nonperturbative structure of the theory could be obtained on the basis of purely perturbative information.

The divergence of the perturbation theory in QCD is far more than a merely technical embarassment, as has recently been emphasized by Mueller [6]. It fundamentally limits our ability to reliably compute important corrections (such as higher twist effects) to the vast phenomenology of high energy processes described by perturbative QCD. Specifically, in those cases where one attempts to weld analytic perturbative with numerical or phenomenological nonperturbative estimates for the same process, the precision of the result is clouded by unavoidable resummation ambiguities on the perturbative side. Consider, for example, a renormalization group controlled amplitude $\Pi\left(Q^{2}\right)$ in QCD, expressible as a formal (divergent asymptotic) series in the running coupling $\alpha\left(Q^{2}\right)$ :

$$
\Pi\left(Q^{2}\right) \simeq \sum_{n} c_{n} \alpha^{n}\left(Q^{2}\right)
$$

Quite generically, one may identify subsets of graphs in perturbation theory which contribute factorial growth to the dimensionless coefficients $c_{n}$ at large $n$

$$
c_{n} \simeq\left(\frac{b_{0}}{p}\right)^{n} n^{\gamma} n !
$$

where $b_{0}, b_{1} \equiv \frac{b_{0}^{2} \gamma}{2}$ are the first two coefficients of the beta function, and $p$ is a positive integer. Using Stirling's approximation, the $n$-th term in the asymptotic series (1) may be written

$$
n !\left(\frac{b_{0}}{p}\right)^{n} n^{\gamma} \alpha\left(Q^{2}\right)^{n} \simeq e^{n \ln \left(n b_{0} \alpha / p\right)-n}
$$


which is minimal at $n_{\min } \simeq \frac{p}{b_{0} \alpha\left(Q^{2}\right)} \simeq p \ln \frac{Q^{2}}{\Lambda^{2}}$. The error in an asymptotic expansion is typically at least as large (it may of course be much larger !) as the smallest term, here

$$
e^{-n_{\min }} \simeq\left(\frac{\Lambda^{2}}{Q^{2}}\right)^{p}
$$

which is power suppressed at large $Q$ in just the way we expect for a higher twist term. The moral is clear: higher twist effects are not precisely calculable in the absence of a reliable resummation procedure. The same arguments in fact apply in a variety of other important situations: for heavy quark expansions, where $1 / M^{p}$ effects ( $M=$ heavy quark mass) may be confused with resummation ambiguities arising in a static-quark quantity evaluated via the renormalization group in terms of a perturbative expansion in $\alpha(M)$, or in estimating analytically power corrections to lattice quantities in the lattice spacing $a$.

The conventional Borel transform approach (for a thorough review, see [7]) attempts to reconstruct the full nonperturbative structure of the theory from knowledge of the behavior of weak-coupling perturbation theory at large orders. There are two serious problems with such an approach in infrared unstable theories like QCD. First, it is becoming increasingly apparent that the large-order behavior is exceedingly complex 8 ]. It is very difficult, even restricting oneself to a strictly limited subset of graphs, to precisely identify the terms which give the truly dominant behavior at large order, due to the subtle interplay of combinatoric and kinematic effects. Secondly, in a non-Borel-summable theory (and any infrared unstable theory with dynamical mass generation falls into this category) there is in any case 
no reliable reconstruction theorem based on the usual perturbative Borel transform, which develops singularities (renormalons) on the positive real axis of the Borel variable $s$, i.e on the Borel integral contour. Fortunately, an alternative formulation of the Borel transform exists, first discussed in QCD by t'Hooft [5], and later by Crutchfield [9] and David [10]. This form of the transform is closely connected to the "naive" perturbative definition in Borel-summable theories, but can be used to give a precise reconstruction theorem in a much wider class of theories. The singularity structure of this transform is unambiguously related to the exact nonperturbative structure of the full theory. But by the same token, this structure is not directly accessible in conventional perturbation theory, but must be studied in an explicitly nonperturbative way.

The primary objective of this paper is to suggest that useful information about the renormalon structure (i.e positive $s$ singularities) in infrared unstable theories can indeed be obtained using nonperturbative techniques (such as the large $\mathrm{N}$ expansion, or lattice theory). In Section 2, we review very quickly the conventional perturbative Borel transform $\hat{B}(s)$, and explain its relation to the generalized transform $B(s)$ and the density of states function $\tilde{B}(s)=\operatorname{disc} B(s)$. A classification of renormalon singularities into those which are already present at finite volume ("Type 1") and those which are only strictly speaking present at infinite volume ("Type 2") is given. The basic interconnections are further illustrated using spin models in 1 and 2 dimensions (the nonlinear sigma model) in Section 3. In Section 4 we explain the perturbative camouflage of renormalon singularity structure in disconnected quantities such as the partition function (vacuum-vacuum am- 
plitude). The status of the first infrared renormalon singularity in QCD (recently raised by Brown, Yaffe and Zhai [11]; see also [12]) is discussed in Section 4. It is shown that certain renormalon singularities of the full Borel function $B(s)$ are immune to infrared cutoffs in ordinary perturbation theory which remove the usual IR renormalons. Moreover, the presence of such a singularity appears to be a generic feature once dynamical mass generation is assumed. In Section 5 we study the sigma model at large $\mathrm{N}$ on the lattice in order to establish the numerical feasibility of extracting renormalon singularities by analytic continuation of nonperturbatively computed (say, by Monte Carlo simulation) Euclidean amplitudes. Section 6 summarizes our conclusions and indicates directions for further study.

\section{Borel Transform Technology}

The archetypal example of a Borel-summable perturbation theory is the nonGaussian integral

$$
\begin{aligned}
Z(f) & \equiv \frac{1}{\sqrt{(f)}} \int_{0}^{+\infty} e^{-\frac{1}{f} V(x)} d x, \quad V(x)=x^{2}+x^{4} \\
& =\int_{0}^{+\infty} e^{-x^{2}-f x^{4}} d x \\
& \simeq \sum_{n=0}^{\infty}(-f)^{n} \frac{\Gamma\left(2 n+\frac{1}{2}\right)}{n !} \equiv \sum c_{n} f^{n+1}
\end{aligned}
$$

Evidently the coefficients $c_{n}$ in the asymptotic expansion of $Z(f)$ for $f \rightarrow 0^{+}$ have the large order behavior $c_{n} \simeq(-1)^{n} 4^{n} n$ ! . Define the "naive" Borel transform

$$
\hat{B}(s) \equiv \sum_{n} \frac{c_{n}}{n !} s^{n}
$$


so that $Z_{0}(f) \equiv \int_{0}^{+\infty} e^{-s / f} \hat{B}(s) d s$ and $Z(f)$ have the same asymptotic expansion for small $f$. In this case the analytic properties of $Z(f)$ are such (see Ref [7] for precise conditions) that $Z_{0}=Z$ and knowledge of the "naive" transform leads to a precise reconstruction of $\mathrm{Z}$ for all $f$. We shall actually be using a more powerful version of the Borel method in most of this paper, which will circumvent the need for theorems of this type whcih certify the preceding procedure. The shortcomings of $\hat{B}(s)$ for non-Borel-summable theories, where the sign oscillation in $c_{n}$ is absent in some uncancelled set of contributions at large order (not necessarily the dominant ones!) are immediately apparent: $\hat{B}(s)$ develops singularities on the positive real $s$ axis, rendering the reconstruction integral ambiguous (if the singularities are nonintegrable). The most straightforward way in which such singularities can arise is illustrated in Fig. 1, where the "action" $V(x)$ possesses a secondary nonperturbative extremum.

To expose the singularity in $\hat{B}(s)$ write 13

$$
\begin{aligned}
Z(f) & =\int e^{-V(x) / f} d x \\
& =\int_{0}^{+\infty} d s e^{-s / f} \int d x \delta(s-V(x)) \\
& =\int_{0}^{+\infty} d s e^{-s / f} \tilde{B}(s)
\end{aligned}
$$

where

$$
\tilde{B}(s) \equiv \int d x \delta(s-V(x))=\sum_{V\left(x_{i}\right)=s} \frac{1}{\left|V^{\prime}\left(x_{i}\right)\right|}
$$

If $V$ has a nontrivial extremum where $V^{\prime}\left(x_{i}\right)=0, s_{i}=V\left(x_{i}\right)>0$, we will have necessarily $B\left(s_{i}\right)=\infty$, a singularity (in this case, an integrable squareroot singularity, as $\tilde{B} \simeq 1 / \sqrt{s-s_{i}}$ ) for positive $s$. To the extent that the 


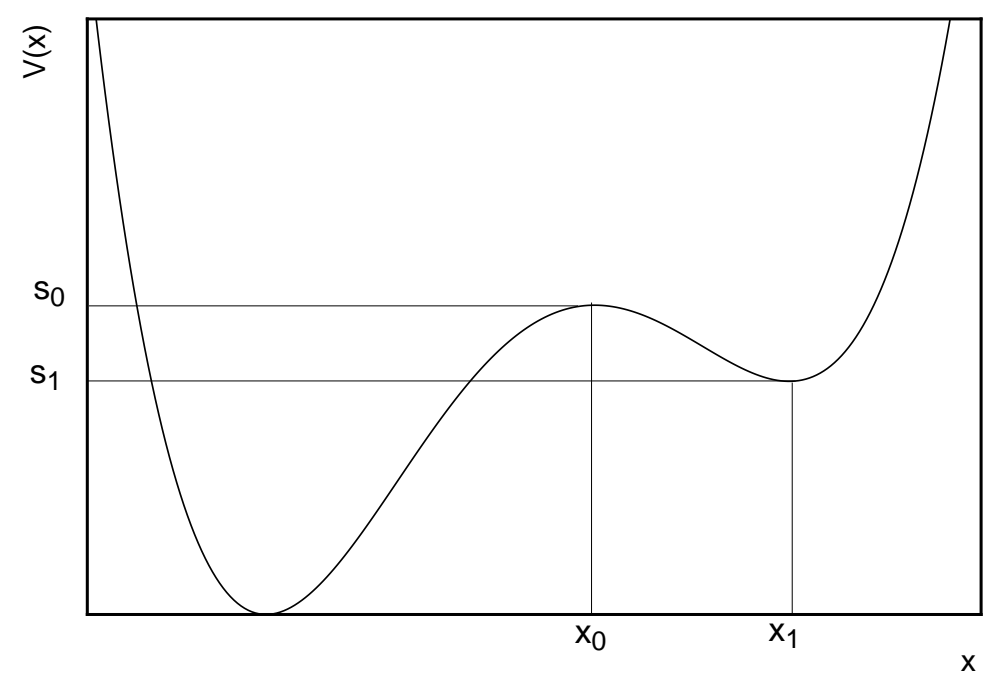

Figure 1: NonGaussian Action leading to singular $\hat{B}(s)$

interchange of integrations carried out in $\mathrm{Eq}(9)$ is legal, the reconstruction of $Z$ from $\tilde{B}$ must be precise. In fact $\tilde{B}$ is intimately related to the more general Borel function to be introduced below. In general, however, $\hat{B} \neq \tilde{B}$ ! Of course, the asymptotic expansions for small $s$ of $\hat{B}, \tilde{B}$, which determine the asymptotic expansion of $Z(f)$ for small $f$, must agree. As pointed out originally by t'Hooft [5], the existence of Euclidean extrema of the action (instantons) in QCD implies in a similar way the presence of singularities in $\tilde{B}(s)$ at $s=S_{\text {inst }}$. Moreover, the location of the singularities (though not their strength) is universal (see ([5]): once a singularity appears in any amplitude of the theory, it is expected to propagate to all others.

For a class of non-Borel-summable theories (the archetype is the double well anharmonic oscillator) an optimized reorganization of perturbation theory can be shown to converge with exponential rapidity 4 to the exact 
partition function. These convergence proofs apply only when the theory is formulated at finite volume, however, limiting the practical usefulness of such methods in higher dimensional field theories. The approach taken here will be to employ a generalized Borel Transform which allows a "bulletproof" reconstruction of the full theory, avoids resummation ambiguities, and is more closely tied to the path integral formulation of the theory [9]. Our ultimate objective is to explore the potential of the presently available nonperturbative lattice techniques for computing the singularity structure of this transform.

Consider a typical infrared-unstable (asymptotically free) theory, such as the $\mathrm{O}(\mathrm{N})$ sigma model in 2 dimensions, or $\mathrm{QCD}$ in 4 dimensions, defined by a functional integral

$$
Z(f)=\int \mathcal{D} \phi e^{-\frac{1}{f} S[\phi]}
$$

where $\phi$ is a generic set of fields, $S[\phi])$ the Euclidean action, and $f$ a bare coupling constant. A compact lattice formulation $\left(\int \mathcal{D} \phi=1\right)$ of the theory is supposed, so that a UV cutoff is always present, and we have logarithmic asymptotic freedom

$$
f \sim \frac{1}{b_{0} \ln (\Lambda / m)}, \quad \Lambda>>m
$$

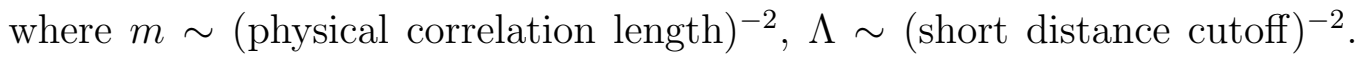
For both sigma models and QCD on a finite lattice, $0 \leq S[\phi] \leq S_{\max }$ is a bounded continuous function of the fields, and the integral $\int \mathcal{D} \phi$ is compact. Define the generalized Borel Transform $B(s)$ as the Laplace transform with respect to inverse coupling, as follows

$$
B(s) \equiv \int_{0}^{+\infty} e^{s / f} Z(f) d\left(\frac{1}{f}\right), \quad \operatorname{Re}(s)<0
$$


The integral is convergent at large $f$ for all $s\left(\right.$ as $e^{s / f} Z(f) \rightarrow 1$ ) and for $\operatorname{Re}(s)<0$ as $f \rightarrow 0^{+}$. Thus $B(s)$ is left-half-plane analytic, with a cut on the positive real axis for $0<s<S_{\max }$. Indeed, inserting the functional integral expression (13) in (15), and defining $x \equiv \frac{1}{f}$,

$$
\begin{aligned}
B(s) & =\int \mathcal{D} \phi \int_{0}^{+\infty} e^{x(s-S[\phi])} d x \\
& =\int \mathcal{D} \phi \frac{1}{S[\phi]-s}
\end{aligned}
$$

which is manifestly analytic in the $s$-plane cut along the positive real axis between $s=0$ and $s=S_{\max }$. The discontinuity of $B(s)$ across this cut is

$$
\operatorname{Im} B\left(s=s_{R}+i \epsilon\right)=\pi \int \mathcal{D} \phi \delta\left(s_{R}-S[\phi]\right) \equiv \pi \tilde{B}\left(s_{R}\right)
$$

The physical interpretation of this discontinuity is clear: $\tilde{B}(s)$ is the density of configurations in the functional integral with action equal to $s$ (cf. Eq(12)). Singularities of $\tilde{B}$ are expected wherever there is a sharp local enhancement in the number of configurations, for example wherever the action has a local extremum.

The rigorous reconstruction of $Z(f)$ from $\tilde{B}$ is trivial in this framework. The vertical contour of the inverse Laplace transform may be wrapped around the cut on the positive $s$ axis to give an integral over the discontinuity of $B(s)$, i.e. over $\tilde{B}$. More directly, we may simply observe that it follows from $\mathrm{Eq}(13,18)$ that

$$
Z(f)=\int_{0}^{+\infty} \tilde{B}(s) e^{-s / f} d s
$$

This reconstruction is exact: there are no routing ambiguities in the $s$ integral. Of course, $\tilde{B}$ is in general a distribution (containing potentially $\delta$ 
singularities), not obtainable purely from knowledge of weak-coupling perturbation theory. Instead, it must be computed nonperturbatively by analytic continuation of $B(s)$, computed nonperturbatively in the region $\operatorname{Re}(s)<0$, where the defining integral (15) exists. It should be noted at this point that $\tilde{B}(s)$, in contrast to $B(s)$ may have singularities in the left-half-plane (corresponding to non-principal-sheet singularities of $B(s)$ ): the ultraviolet renormalons of the renormalized perturbation theory in QCD are of this type.

It is convenient to distinguish between two types of singularities appearing in $\tilde{B}(s)$. Exact local extrema of the Euclidean action may exist even for systems with a finite number of degrees of freedom (e.g. field theories formulated on a finite lattice). Such extrema give rise to actual singularities (henceforth called "Type 1") of $\tilde{B}$ even at finite volume, when the theory is cutoff in the infrared. For example, one can find lattice analogs of the instanton solutions of the classical 2D O(3) sigma model using a simple numerical annealing procedure to solve the lattice Euclidean field equations. Other singularities of $\tilde{B}$ only appear at infinite volume, and will be called Type 2 singularities. The famous infrared renormalons of large order perturbation theory are connected to singularities of this type. As numerical simulations of nonperturbative behavior are necessarily restricted to finite volume lattice theories, the question arises whether singularities of this type are really accessible using simulation techniques. We shall see below that the question is complicated by the nonuniformity of the analytic continuation to positive $s$ in the large volume limit, which will require a modification of the definition (15) in the finite volume situation. For the time being, let us illustrate the absence of a true singularity in (15) in a discrete system with a simple toy 
model. Consider a mass gap relation connecting a bare coupling $f$ and a dynamically generated mass $m$ of the form

$$
\frac{1}{f}=\frac{1}{L} \sum_{n=0}^{L} \frac{1}{m+n / L} \rightarrow \ln \left(\frac{1+m}{m}\right), L \rightarrow \infty
$$

The "infinite volume limit" thus gives $m=\frac{e^{-1 / f}}{1-e^{-1 / f}}$, and Borel transforms $B(s)$ of any analytic function $G(m)$ expandable at $m=0$ will display simple poles at $s=0,1,2,3 \ldots$ For example, if $G(m)=\frac{1}{1+m}$, we have (at $L=$ क) $B(s)=-1 / s-1 /(1-s)$ (for other choices of the "momentum" in the denominator of $G(m)$, there will typically be poles at all integer $s$ ). On the other hand, for finite $L$, we have

$$
\begin{aligned}
B_{L}(s) & =\int_{0}^{\infty} e^{s / f} \frac{1}{1+m} d\left(\frac{1}{f}\right) \\
& =\int_{0}^{\infty} \frac{1}{L} \sum \frac{1}{(m+n / L)^{2}} \frac{1}{1+m} e^{s \cdot \frac{1}{L} \sum \frac{1}{m+n / L}} d m
\end{aligned}
$$

where the $m$-integral in (21) is convergent for $\operatorname{Re}(s)<0$. We may analytically continue to $s=1$ by deforming the $m$ contour to avoid a singularity from the $n=0$ term in the exponent,i.e. by keeping $\arg \left(\frac{s}{m}\right)$ fixed near $m=0$. This can be done without encountering any pinches of the integration contour. Consequently, $B_{L}(s=1)$ is perfectly finite for finite $L$. In Section 6 we shall see that $B_{L}(s)$ needs to be modified so that a smooth approach to the infinite volume behavior for positive $s$ is obtained.

\section{Non-Borel-summable spin models}




\subsection{Separable models $(\mathrm{D}=1)$}

A simple model which already serves to illustrate several features of the renormalon singularity structure in more general non-Borel theories is the 1D spin chain defined by the action

$$
S=\sum_{i=1}^{L}\left(1-\hat{\phi}_{i} \cdot \hat{\phi}_{i+1}\right)
$$

Here the $\hat{\phi}_{i}$ are unit 3-vectors and free boundary conditions are chosen so that the model factorizes in a trivial way. Indeed, the partition function is

$$
\begin{aligned}
Z_{L}(f) & =\int \Pi_{i} d \hat{\phi}_{i} e^{-S[\hat{\phi}] / f} \\
& =\left(\frac{f}{2}\right)^{L}\left(1-e^{-2 / f}\right)^{L}
\end{aligned}
$$

showing a trivial separation of the "perturbative" $\left(\propto f^{L}\right)$ and "nonperturbative" $\left(\propto f^{L} e^{-2 n / f}\right)$ terms. For $L=1$, the Borel Transform of $Z$ is

$$
B_{1}(s)=\frac{1}{2} \ln \left(\frac{2-s}{-s}\right)
$$

with discontinuity

$$
\tilde{B}_{1}(s) \equiv \frac{1}{\pi} \operatorname{Im} B(s+i \epsilon)=\frac{1}{2} \theta(s) \theta(2-s)
$$

Note that poles only appear in $\tilde{B}_{1}(s)$ after a derivative with respect to $s$. This effectively (cf. Eq(15)) removes the overall perturbative factor of $f$ (associated with the measure in the path integral), exposing the nonperturbative behavior. For $L$ large, $\tilde{B}_{L}(s)$ is even smoother (each prefactor of $f$ effectively integrates with respect to $s$ ). The singularity structure is numerically invisible in this case. In Fig. 2 we show $\tilde{B}_{8}(s)$. It is clearly difficult to tell 
that there are actually singularities at $s=2,4,6,8, \ldots$ ! In Section 4 we shall see that this perturbative camouflaging of the singularity structure is typical of disconnected quantities like $Z$, but is not a problem when connected quantities (with a well-defined infinite volume limit) are considered.

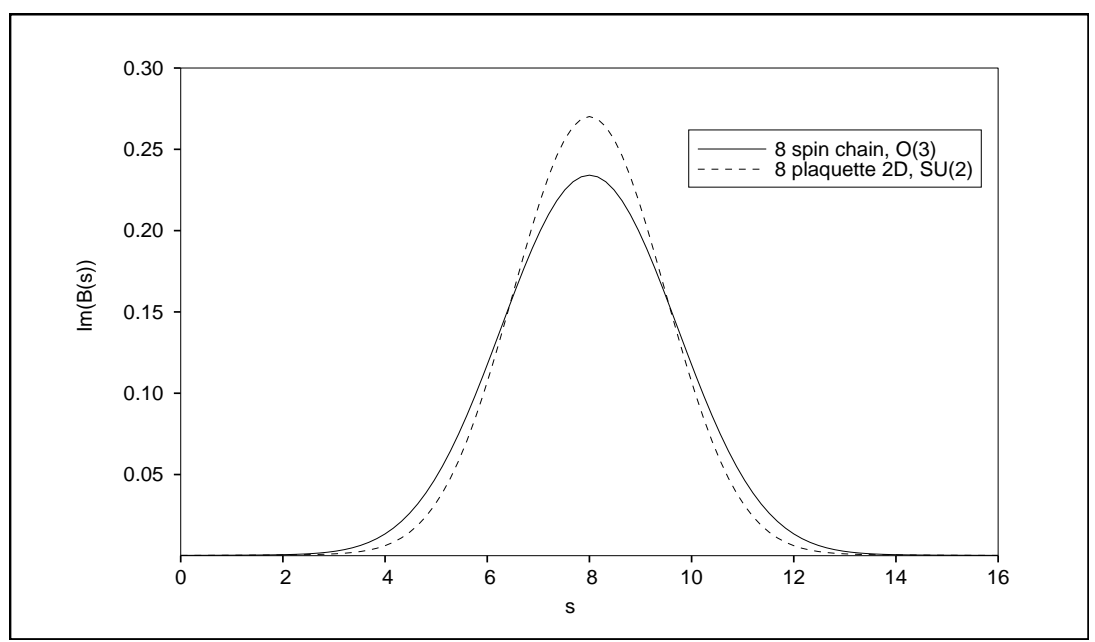

Figure 2: Borel Transform $\tilde{B}$ for $L=8$ spin chain

In agreement with the usual analogy between spin models in D and gauge models in 2D dimensions, the separable model of 2D pure lattice gauge theory is found to have an essentially identical Borel structure. If we take the gauge group to be $\mathrm{SU}(2)$, the partition function for a system of $L$ plaquettes (with free boundary conditions) is found to be $Z_{L}(f)=\left[2 f e^{-1 / f} I_{1}\left(\frac{1}{f}\right)\right]^{L}$. For $L=1$, the Borel Transform is $\tilde{B}_{1}(s)=\sqrt{s(2-s)}$. The Borel discontinuity (=density of states in functional integral) for a system of 8 plaquettes is also plotted in Fig(2) for comparison with the spin chain case. Once again, the singularities at $s=2,4,6, \ldots$ are numerically invisible. 


\subsection{Borel Structure of 2D Sigma model}

The partition function of the continuum 2D Sigma model is given by the Euclidean functional integral

$$
Z(f)=\int \mathcal{D} \hat{\phi}(x) e^{-\frac{1}{2 f} \int d^{2} x\left|\partial_{\mu} \hat{\phi}\right|^{2}}
$$

Here $\hat{\phi}$ is a unit N-vector field, $f$ is a bare coupling, and a UV cutoff is presumed. On a finite 2 dimensional $L x L$ lattice, the corresponding quantity is

$$
\begin{aligned}
Z_{L}(f) & =\int \Pi_{n} d \vec{\phi}_{n} d \rho_{n} e^{i \sum_{n} \rho_{n}\left(\vec{\phi}_{n}^{2}-1\right)-\frac{N}{2 f} \sum_{n, \mu}\left|\Delta_{\mu} \vec{\phi}_{n}\right|^{2}} \\
& =\left(\frac{f}{N}\right)^{N L^{2}} \int \Pi_{n} d \vec{\phi}_{n} d \rho_{n} e^{i \sum_{n} \rho_{n}\left(\vec{\phi}_{n}^{2}-N / f\right)-\frac{1}{2} \sum_{n, \mu}\left|\Delta_{\mu} \vec{\phi}_{n}\right|^{2}}
\end{aligned}
$$

Here $n$ labels lattice sites, and an auxiliary condensate field $\rho_{n}$ has been introduced to implement the nonlinear constraint. Integrating out the $\vec{\phi}$ fields

$$
Z_{L}(f)=\left(\frac{f}{N}\right)^{N L^{2}} \int \Pi_{n} d \rho_{n} e^{-\frac{N}{2} S_{\mathrm{eff}}\left[\rho_{n}\right]}
$$

where

$$
S_{\text {eff }}=\operatorname{Tr} \operatorname{Ln}\left(-\Delta^{2}-2 i \rho\right)+\frac{2 i}{f} \sum_{n} \rho_{n}
$$

For large $N$, the functional integral in $\mathrm{Eq}(28)$ is dominated by a translationally invariant complex saddle point at $\rho_{n}=\rho_{\text {sad }}$ where

$$
\frac{1}{f}=\frac{1}{L^{2}} \sum_{\vec{k}} \frac{1}{d(\vec{k})+m}, \quad m \equiv-2 i \rho_{\mathrm{sad}}
$$

Here the kinematic momentum factor appearing in the propagator denominator is $d(\vec{k}) \equiv 4\left(\sin \left(\pi k_{x} / L\right)^{2}+\sin \left(\pi k_{y} / L\right)^{2}\right)$. Thus the $1 / N$ expansion 14 
generates immediately a dynamical (squared) mass $m(f)$. Note that for any finite $L$, the dynamical mass $m(f)$ defined implicitly in Eq(30) is actually analytic at $f=0$. Any analytic function of $m$ (e.g. the large $N$ limit of the $\phi$ propagator at momentum $\vec{k}$, namely $\left.\frac{1}{d(\vec{k})+m}\right)$ will therefore have a conventional Borel transform $\hat{B}(s)$ which is entire in $s$. The full Borel transform $B(s)$ of the large $N$ propagator has a cut on the positive axis, but no true singularities of $\tilde{B}$ for finite $L$. The problem of recovering the singularities of the large volume limit from lattice data will be discussed in Section 6 . Type 1 (instanton) singularities which would surface already at finite $L$ are of course absent in the large $N$ limit.

The large $N$ result for the partition function is

$$
Z_{L} \simeq(f / N)^{N L^{2}} e^{-\frac{N L^{2}}{2}\left(\frac{1}{L^{2}} \sum_{\vec{k}} \ln (d(\vec{k})+m)-m / f\right)}
$$

Again, as for the spin chain case, the perturbative prefactor will numerically camouflage the nonperturbative structure.

For future reference, we list here several important features of the large $N$ limit in the continuum theory. Initially, assume both infrared and ultraviolet cutoffs to be present for the modes of the $\vec{\phi}$ field: $\lambda<k^{2}<\Lambda$. The mass gap equation then becomes

$$
\begin{aligned}
\frac{1}{f} & =\int \frac{d^{2} k}{(2 \pi)^{2}} \frac{1}{k^{2}+m} \\
& =\frac{1}{4 \pi} \ln \left(\frac{\Lambda+m}{\lambda+m}\right)
\end{aligned}
$$

giving a dynamical squared mass

$$
m=\frac{\Lambda e^{-4 \pi / f}-\lambda}{1-e^{-4 \pi / f}}
$$


displaying the characteristic essential singularity at $f=0$. The singularity is present even though $\lambda \neq 0$ (and conventional perturbative IR renormalons are absent:cf. Section 5)! The large $N$ limit of the $\vec{\phi}$ propagator in momentum space

$$
\frac{1}{q^{2}+m}=\frac{1}{q^{2}}-\frac{m}{q^{4}}+\frac{m^{2}}{q^{6}}-. .
$$

possesses a meromorphic Borel transform $B(s)$. Taking $\lambda=0$ for simplicity and changing variables to $x \equiv 1 / f$

$$
\int_{0}^{+\infty} d x e^{s x}\left(\frac{\Lambda e^{-4 \pi x}}{1-e^{-4 \pi x}}\right)^{n}=\Lambda^{n}\left(\frac{1}{4 \pi n-s}+\frac{n}{4 \pi(n+1)-s}+. .\right)
$$

so there are simple poles at $s=4 \pi, 8 \pi, 12 \pi, \ldots$ Switching on the infrared cutoff $\lambda$ modifies the residue, but not the location of these poles. This apparently paradoxical situation is elucidated further in Section 5.

The meromorphic character of the Borel transform of the propagator extends to other Green's functions in the large $N$ limit. The two-current correlator, for example, is defined by

$$
\begin{aligned}
\Pi^{\mu \nu}(q) & =\frac{1}{N^{2}} \int d^{2} x<0\left|J_{a b}^{\mu}(x / 2) J_{a b}^{\nu}(-x / 2)\right| 0>e^{-i q \cdot x} \\
J_{a b}^{\mu} & \equiv \frac{1}{2} \phi_{a} \partial^{\mu} \phi_{b}
\end{aligned}
$$

Neglecting terms of $O\left(q^{2} / \Lambda\right)$ and subtracting at $q=0$, one has (in the large N limit)

$$
\Pi^{\mu \nu}=-\frac{1}{8 \pi}\left(g^{\mu \nu}-\frac{q^{\mu} q^{\nu}}{q^{2}}\right) \int_{0}^{1} d x \ln \left(1+x(1-x) \frac{q^{2}}{m}\right)
$$

The Borel transform can be computed by leaving the Feynman parameter integration to the end. Again neglecting terms of order $q^{2} / \Lambda$ (but not $m / q^{2}$ !) 
the relation between $m$ and $f$ is effectively $m=\Lambda e^{-4 \pi / f}$ so

$$
B^{\mu \nu}(s)=-\frac{1}{32 \pi^{2}} \Lambda^{s / 4 \pi}\left(g^{\mu \nu}-\frac{q^{\mu} q^{\nu}}{q^{2}}\right) \int_{0}^{1} d x \int_{0}^{\Lambda} d m m^{-1-s / 4 \pi} \ln \left(1+\frac{x(1-x) q^{2}}{m}\right)
$$

We are interested in the singularity structure of $B^{\mu \nu}(s)$ for $\operatorname{Re}(s)>0$ (the generalized Borel Transform is left-half-plane analytic). Since

$$
\int_{0}^{1} d x \int_{\Lambda}^{+\infty} d m m^{-1-s / 4 \pi} \ln \left(1+\frac{x(1-x) q^{2}}{m}\right)
$$

is analytic for $\operatorname{Re}(s)>0$, we may complete the $m$ integral to $\int_{0}^{+\infty}$ without altering the singularity structure in the right-half-plane. One then finds

$$
B^{\mu \nu}(s ; q) \simeq \frac{1}{32 \pi^{2}}\left(g^{\mu \nu}-\frac{q^{\mu} q^{\nu}}{q^{2}}\right)\left(\frac{\Lambda}{q^{2}}\right)^{s / 4 \pi} \frac{\Gamma(s / 4 \pi) \Gamma(-s / 4 \pi) \Gamma(1-s / 4 \pi)^{2}}{\Gamma(2-s / 2 \pi)}
$$

which has double poles at $s=4 \pi, 8 \pi, \ldots$ These poles would be visible as sharp peaks if $B^{\mu \nu}(s)$ could be computed at $\operatorname{Re}(s)<0$ and then analytically continued to $s=s_{R}+i \gamma, \gamma<<s_{R}, s_{R}>0$. Note that $B^{\mu \nu}(s)$ satisfies the Brown-Yaffe-Zhai relation [11]

$$
\operatorname{disc} B^{\mu \nu}\left(s ; q^{2}=-Q^{2}\right)=\sin \left(\pi b_{0} s\right) B^{\mu \nu}\left(s ; Q^{2}\right), \quad b_{0}=\frac{1}{4 \pi}
$$

From the foregoing, it is apparent that the nonlinear sigma-model in the large $N$ limit exhibits an interesting and elaborate renormalon pole structure [10] in the right-half Borel plane. This is so even though a conventional perturbation expansion applied to the large $N$ result leads to trivial results, insofar as the nonperturbative structure enters in this limit entirely through the quantity $m \sim e^{-4 \pi / f}$ which vanishes to all orders in a formal expansion around $f=0$. 


\section{Perturbative Camouflage of Renormalon Sin- gularities}

Formally the most convenient field-theoretic quantity from the point of view of the generalized Borel function $B(s)$ is the basic partition function $Z(f)$ of $\mathrm{Eq}(13)$, as the discontinuity of $B(s)$ in this case is nothing but the density of configurations in a given action shell in the functional integral. Unfortunately, $Z$ does not possess an infinite volume limit, and as we saw in Section 3 , the Type 1 singularities of $B(s)$ for a system of large but finite volume $V$ are in any event camouflaged by perturbative prefactors $\sim f^{V}$ which smooth the $s$-dependence (as multiplication by $f$ is equivalent to integration by $s$ ). The Borel transform of $Z(f)$ is very accurately computable by Monte Carlo techniques: from the density of states interpretation described in Section 2 one has only to histogram the frequency of configurations generated in a Monte Carlo simulation (relative to a conveniently chosen reference action) versus action. The result of a simulation of $\tilde{B}(s)$ for a $8 \times 82 \mathrm{D}$ sigma model is shown in Fig(3), to be compared with Fig(2) for the spin chain or 2D QCD. Note that the singularities at positive $s$ are numerically invisible.

The connected vacuum amplitude $W(f) \equiv \ln Z / V$ is clearly preferable to $Z$ in this regard. Apart from having a well-defined infinite volume limit, perturbative prefactors in $Z$ appear as additive contributions in $W$, effectively uncovering the renormalon structure of the theory. We may expect the same

to be true of other connected Green's functions (e.g. two-point functions) of the theory, which are expressible as source-derivatives of $W$. For example, 
consider the trivial spin-chain model of Section (3.1):

$$
W_{L}=\frac{1}{L} \ln Z_{L}=\ln (f / 2)+\ln \left(1-e^{-2 / f}\right)
$$

the Borel transform $B_{W}$ of which exhibits simple poles at $s=2 n, n=1,2,$. . with residue $1 / n$. The continuation to positive $s$ thus exhibits Lorentzian peaks:

$$
\begin{aligned}
B_{W}(s) & \sim \sum_{n} \frac{1}{n} \frac{1}{s-n} \\
\operatorname{Im} B_{W}\left(s_{R}+i \gamma\right) & \sim \gamma \sum \frac{1}{n} \frac{1}{\left(s_{R}-n\right)^{2}+\gamma^{2}}
\end{aligned}
$$

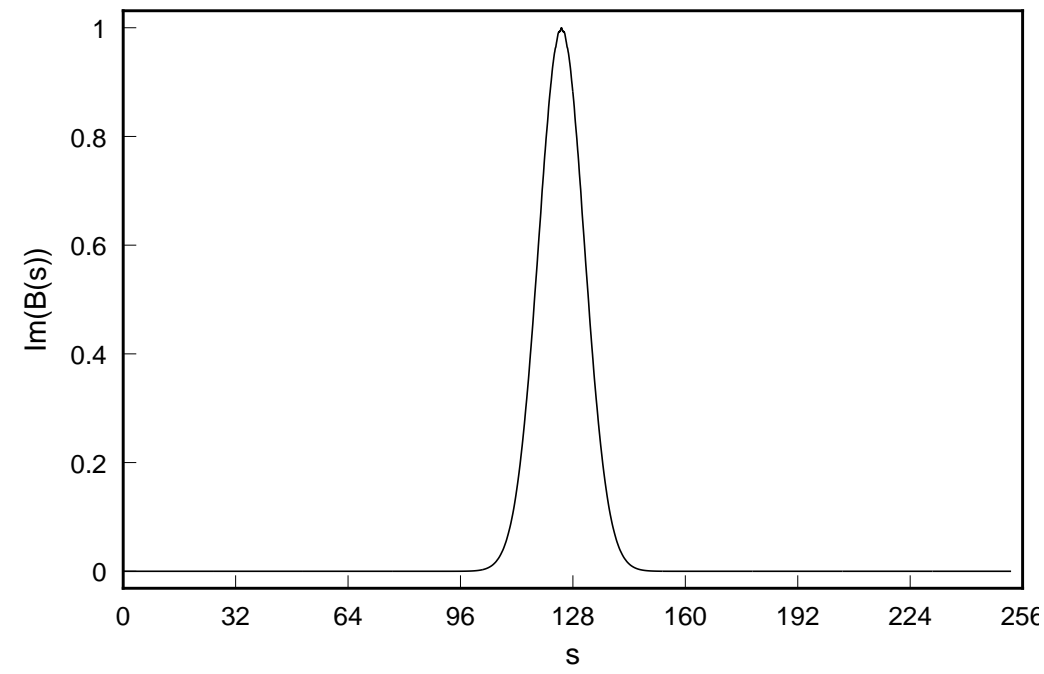

Figure 3: Density of configurations for 2D O(3) Sigma model

Assuming the Borel discontinuity $\tilde{B}(s)$ of $Z$ has been computed (say, by Monte Carlo) sufficiently accurately, the analytic continuation of $Z(f)$ to $x \equiv \frac{1}{f}=i y$ ( $y$ positive real) is available as the Fourier transform of $\tilde{B}$. Recall

$$
Z(x=1 / f)=\int_{0}^{+\infty} d s \tilde{B}(s) e^{s x}
$$


while

$$
B_{W}(s) \equiv \int_{0}^{+\infty} d x e^{s x} \ln Z(x), \quad \operatorname{Re}(s)<0
$$

Analytically continue $B_{W}$ from real negative $s$ to $s=s_{R}+i \gamma\left(s_{R}, \gamma>0\right)$ by simultaneously deforming the $\mathrm{x}$-contour in $\mathrm{Eq}(45)$ to $x=i y$. Thus

$$
\operatorname{Im} B_{W}\left(s_{R}+i \gamma\right)=\operatorname{Re} \int_{0}^{\infty} d y e^{i s_{R} y-\gamma y} \ln \int_{0}^{S_{\max }} d s \tilde{B}(s) e^{i s y}
$$

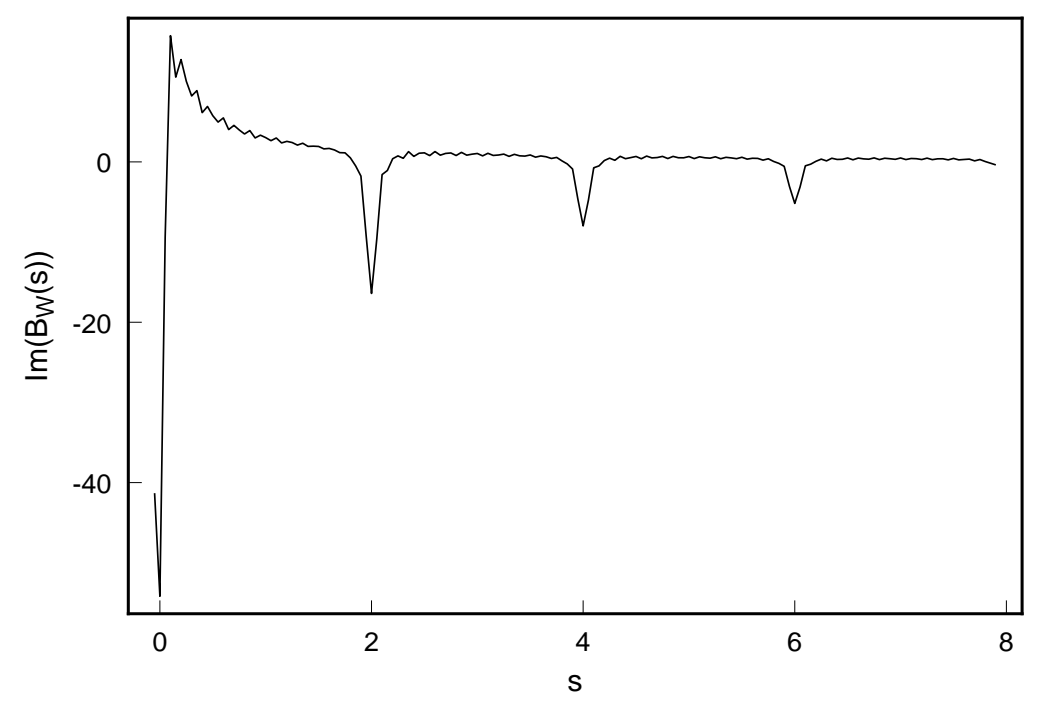

Figure 4: Borel singularities of $W$ in $1 \mathrm{D} \mathrm{O}(3)$ spin chain

The result of this computation for the spin chain model is shown in Fig(泪) for $\gamma=0.05$. The singularities at $s=2,4,6, .$. are clearly visible. (The oscillatory structure visible in Fig(田) is due to the fact that the integral over $y$, performed numerically, was cut off at the upper end). It would be clearly of great interest to locate the Type 1 singularities in more interesting lattice field theories along these lines. At present most of the discussion of renormalon 
structure centers on correlation functions (e.g. the Euclidean current-current correlator) and we shall focus instead on an alternative approach which allows the extraction of renormalon singularities (of both types) of connected Green's functions.

\section{Status of the First Infrared Renormalon in QCD}

It has been known for some time that evidence for a nontrivial singularity structure in the right-half-plane of the Borel variable $s$ can be detected within the framework of conventional (weak-coupling) perturbation theory. Consider an asymptotically free field theory (such as massless QCD) with no intrinsic mass scale at the classical level. Any scalar Euclidean momentum space correlation function $\Pi\left(Q^{2}\right)$ corresponding to a renormalization group invariant quantity will have a formal perturbative expansion in powers of the running coupling $\alpha\left(Q^{2}\right)$ :

$$
\Pi\left(Q^{2}\right) \sim \sum_{n} c_{n} \alpha^{n}\left(Q^{2}\right)
$$

An example of great phenomenological interest is the hadronic electromag-

netic current correlator, whence $\Pi\left(Q^{2}\right)=\frac{1}{3 Q^{2}} \int d^{4} x e^{i q \cdot x}<0\left|T\left[j^{\mu}(x) j_{\mu}(0)\right]\right| 0>$. Perturbative infrared renormalons are associated with subsets of graphs which result in a contribution to the coefficients $c_{n}$ at large $n$ of the form

$$
c_{n} \sim n !\left(\frac{b_{0}}{p}\right)^{n}
$$


where $b_{0}=\frac{11-2 N_{f} / 3}{4 \pi}$ is the first nontrivial coefficient in the beta-function and $p$ is an integer. The behavior (48) implies a singularity in the naive Borel Transform $\hat{B}(s)$ at $s=p / b_{0}$. Such terms can be seen to arise from sets of graphs generating the leading infrared logarithms in the current-current correlator (see Fig(5)). Introducing an infrared cutoff $\lambda$, the contribution of the subset of graphs shown in Fig(5) is found to be [6]

$$
\int_{\lambda^{2}} l^{2} d l^{2} \frac{\alpha\left(Q^{2}\right)}{1+b_{0} \alpha\left(Q^{2}\right) \ln \left(\frac{l^{2}}{Q^{2}}\right)} \sim \sum \alpha^{n+1}\left(Q^{2}\right)\left(b_{0}\right)^{n} \int_{c}^{\ln \left(Q^{2} / \lambda^{2}\right)} x^{n} e^{-2 x} d x
$$

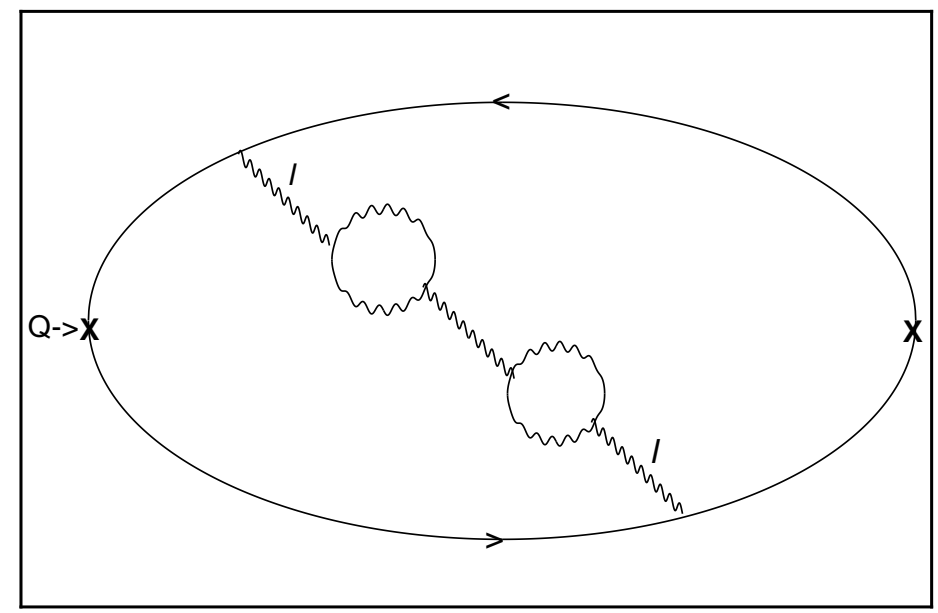

Figure 5: One of a set of bubble graphs contributing to the leading perturbative renormalon in $\mathrm{QCD}$

If no infrared cutoff is present $(\lambda=0)$, the large $n$ behavior of the $x$ 
integral is dominated by $x \sim n$ and we have

$$
c_{n} \sim\left(\frac{b_{0}}{2}\right)^{n} n !
$$

implying a singularity in $\hat{B}(s)$ at $s=\frac{2}{b_{0}}$. The absence of a singularity at the first possible renormalon location $s=\frac{1}{b_{0}}$ can be related to the absence of a local gauge-invariant operator of dimension 2 in QCD (the extra factor of $l^{2}$ in the numerator of (49) is a direct consequence of gauge-invariance). The nonperturbative ambiguity associated with the singularity at $s=\frac{2}{b_{0}}$ is of the form $e^{-\frac{2}{b_{0}} \alpha\left(Q^{2}\right)} \sim \frac{1}{Q^{4}}$, exactly the leading power behavior associated with coefficient functions of the lowest dimension nontrivial operator $F_{\mu \nu}^{2}$ appearing in the operator product expansion of this current correlator.

With an infrared cutoff present, the renormalon singularity disappears, as the large $\mathrm{n}$ behavior is dominated by $x \sim \ln \left(\frac{Q^{2}}{\lambda^{2}}\right)$ and

$$
c_{n} \sim \ln \left(\frac{Q^{2}}{\lambda^{2}}\right)^{n}
$$

This power growth means that the contribution from these graphs to $\hat{B}(s)$ is entire in $s$. Of course, the Taylor series generated by the coefficients (51) has a finite radius of convergence (before the Borel transform). The divergence occurring when $\lambda$ is decreased to the point where $\ln \left(Q^{2} / \lambda^{2}\right) \sim \frac{1}{b_{0} \alpha\left(Q^{2}\right)}$ is just the entrance of the Landau singularity into the range of the momentum integration.

The preceding discussion applies almost word for word to the two current correlator (36) in the 2D sigma model. A typical set of diagrams giving an infrared renormalon in this theory is shown in Fig(6). Here the current 
insertions do not decouple at zero momentum and we have, instead of (49),

$$
\int_{\lambda^{2}} d l^{2} \frac{\alpha\left(Q^{2}\right)}{1+b_{0} \alpha\left(Q^{2}\right) \ln \left(\frac{l^{2}}{Q^{2}}\right)} \sim \sum \alpha^{n+1}\left(Q^{2}\right) b_{0}^{n} \int^{\ln \left(\frac{Q^{2}}{\lambda^{2}}\right)} x^{n} e^{-x} d x
$$

If the infrared cutoff is set to zero, the large order behavior is $c_{n} \sim b_{0}^{n} n$ !, leading to a singularity in $\hat{B}(s)$ at $s=\frac{1}{b_{0}}$. This corresponds to a local operator $\left|\partial_{\mu} \vec{\phi}\right|^{2}$ of dimension 2. If $\lambda \neq 0$, the perturbative renormalon disappears completely, as the large order growth is power rather than factorial.

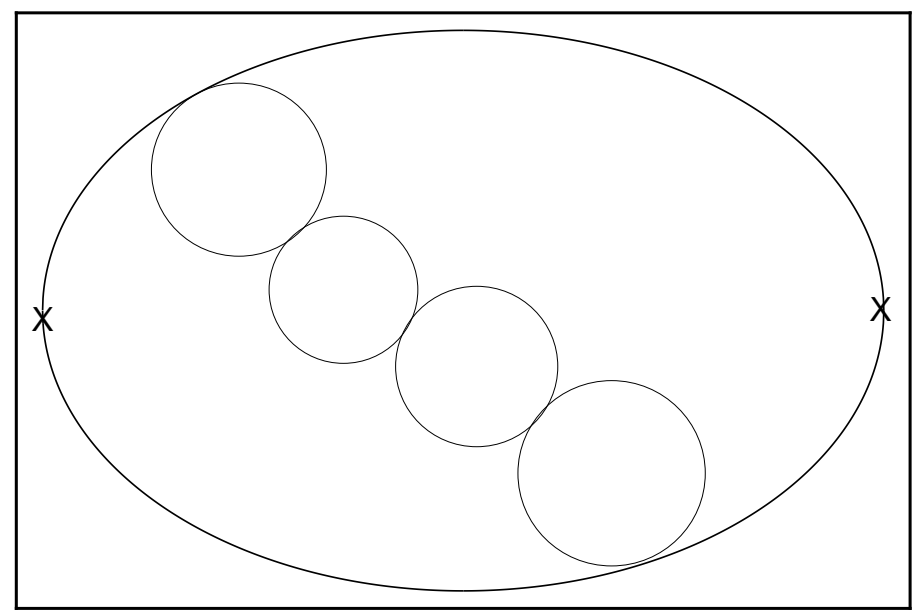

Figure 6: One of a set of bubble graphs contributing to the leading perturbative renormalon in the $2 \mathrm{D}$ sigma model

An important advantage of the 2D sigma model is the availability of the large $N$ saddle-point technique as a reliable tool for exhibiting the mechanism of dynamical mass generation in the theory. It was pointed out previously that dynamical mass generation occurs even if an infrared cutoff is imposed 
on the modes of the elementary $\vec{\phi}$ fields of the theory (recall (33), where $m, \Lambda, \lambda$ represent the squared mass, UV and IR cutoffs resp.). From (33) it is apparent that even if $\lambda \neq 0$, the Borel Transform $B(s)$ of the propagator $\frac{1}{q^{2}+m}$ has simple poles at $s=4 \pi n$, just as in (35). However, we have just seen that if $\lambda \neq 0$, the usual perturbative sources of infrared renormalon singularities in the naive transform $\hat{B}(s)$ disappear! In fact, the renormalon structure of the full Borel function $B(s)$ in this case is associated with the appearance of a zero-momentum condensate for the auxiliary $\mathrm{O}(\mathrm{N})$ singlet field $\rho(x)$ (see (27)), which couples to the singlet two $\phi$ channel. Even though the individual $\vec{\phi}$ constituents are restricted to have nonzero momentum $k^{2}>\lambda$, pairs of these quanta can condense at zero momentum, precisely as in the case of Cooper pairs in BCS superconductivity. The moral of this example is clear: the full Borel transform $B(s)$ can have singularities of purely nonperturbative origin, not associated (at least in any direct fashion) with the usual IR renormalons of perturbation theory.

The preceding discussion applies to the renormalon structure of the continuum field theory in which the momentum modes are still continuous, but a sharp infrared cutoff is introduced. The introduction of a finite lattice imposes an infrared cutoff uniformly on all fields of the theory, including the condensate field $\rho$, thereby eliminating the singularities at $s=4 \pi n$ of $B(s)$.

It was pointed out in section 2 that a singularity in $\tilde{B}(s)$ for the Borel transform of the partition function $Z(f)$ is directly related to a singularity in the density of configurations in the defining functional integral for $Z$. Consider the current-current correlator in quenched QCD (i.e. dropping internal quark loops). The UV cutoff will be implemented on a lattice (with 
a bare coupling $\left.\alpha \equiv g_{0}^{2} / 4 \pi\right)$ and the compact link integrations written $\int \mathcal{D} A_{\mu}$ for simplicity. Then (again, representing the lattice Fourier transform in continuum notation for simplicity):

$$
\begin{aligned}
\Pi\left(Q^{2}\right) & =N\left(Q^{2} ; f\right) / Z(f) \\
N\left(Q^{2} ; f\right) & \equiv \frac{1}{3 Q^{2}} \int d^{4} x e^{i q \cdot x} \int \mathcal{D} A_{\mu} \operatorname{Tr}\left[\gamma^{\mu} \Delta\left(x ; A_{\mu}\right) \gamma_{\mu} \Delta\left(-x ; A_{\mu}\right)\right] e^{-\frac{1}{\alpha} S[A]} \\
& =\int \mathcal{D} A_{\mu} \mathcal{P}[A ; q] e^{-\frac{1}{\alpha} S[A]}
\end{aligned}
$$

where $\Delta(x ; A)$ is the quark propagator in the background field $A$. The Borel discontinuity of $N\left(Q^{2} ; f\right)$ is

$$
\begin{aligned}
\tilde{B}_{N}\left(s ; Q^{2}\right) & =\int \mathcal{D} A_{\mu} \mathcal{P}[A ; q] \delta\left(s-S\left[A_{\mu}\right]\right) \\
& =\overline{\mathcal{P}}(s ; q) \tilde{B}_{Z}(s), \quad \tilde{B}_{Z}(s) \equiv \int \mathcal{D} A_{\mu} \delta\left(s-S\left[A_{\mu}\right]\right)
\end{aligned}
$$

Here $\overline{\mathcal{P}}\left(s ; q^{2}\right)$ is the average of $\mathcal{P}\left[A_{\mu} ; q\right]$ over all configurations with action equal to $s . \tilde{B}_{Z}(s)$ is the fundamental density of configurations in the theory. As pointed out by t'Hooft in his seminal study of the renormalon structure of QCD [5], Borel singularities in the transform of $N(f)$ or $Z(f)$ will generically transfer, via the convolution theorem, to the Borel transform of the connected ratio $N / Z$, barring some miraculous cancellation. In fact, the location of renormalon singularities (though not their type and strength) are expected to be substantially independent of the specific Green's function under consideration: rather, they derive from the basic structure of the functional configuration space of the theory.

It has sometimes been asserted that the presence or absence of renormalon singularities can be inferred solely from the assumption of nonperturbative 
validity of the Wilson operator product expansion. For example, the absence of a perturbative IR renormalon in $\hat{B}(s)$ at $s=1 / b_{0}$ was related above to the absence of local gauge-invariant operators of dimension 2. Such a singularity will never be visible in dimensionally renormalized massless perturbative QCD, where the only mass scale in the theory is introduced via the regularization procedure in such a way as to result in purely logarithmic (rather than power) contributions to renormalized quantities. Rather, we are concerned here with intrinsically nonperturbative contributions to the coefficient function $C_{1}(x)$ of the identity operator, which is present regardless of the subsequent set of nontrivial operators in the theory. Such contributions, of the form $m x^{2}$ in coordinate space, where $m$ is a dynamically generated squared mass of the form $\sim e^{-\frac{1}{b_{0} \alpha}}, \quad \alpha \rightarrow 0$, inevitably produce a Borel singularity at the first location $s=\frac{1}{b_{0}}$. On the other hand, they vanish formally to all orders in a weak coupling expansion, and (in a non-Borel theory) are not necessarily reconstructible from conventional sources of factorial growth in perturbation theory (cf. situation discussed above for 2D sigma model in presence of an IR cutoff!).

Finally, let us note that the presumed existence of a mass gap in the theory can be viewed as prima facie evidence for a universal infrared renormalon singularity at the first available location. It is very difficult to see how a singularity at $s=1 / b_{0}$ can be avoided in almost any physical quantity in QCD once such a mass gap is assumed, together with the renormalization group and asymptotic freedom. As the simplest example, consider the finite temperature partition function of pure QCD at low temperature $\beta^{-1}$. Evidently, for large $\beta$, the one glueball sector dominates and (defining 


$$
\begin{aligned}
\left.m \equiv(\text { glueball }- \text { mass })^{2}\right) & \\
Z_{\beta} & =1+V \int \frac{d^{3} p}{(2 \pi)^{3}} e^{-\beta \sqrt{\left(p^{2}+m\right)}}+. . \\
m & =\Lambda e^{-\frac{1}{b_{0} \alpha}}
\end{aligned}
$$

The higher order terms are suppressed at low temperature, so cannot be expected to cancel (except accidentally) any renormalon singularity found in the first nontrivial term. The Borel transform $B_{1}(s)$ of the 1-glueball contribution to the free energy $\ln Z / V$ thus becomes

$$
B_{1}(s)=\int_{0}^{c} d m m^{-1-b_{0} s} \int \frac{d^{3} p}{(2 \pi)^{3}} e^{-\beta \sqrt{\left(p^{2}+m\right)}}
$$

Integrating by parts to expose the pole at $s=\frac{1}{b_{0}}$ we find

$$
B_{1}(s) \sim \frac{1}{1-b_{0} s} \int \frac{d^{3} p}{(2 \pi)^{3}} \frac{\beta e^{-\beta p}}{2 p}
$$

A similar singularity is expected to arise in all connected Green's functions of the theory once dynamical mass generation occurs.

Of course, to settle this contentious issue definitively, it will be necessary to perform reliable, fully nonperturbative calculations of the generalized Borel function $B(s)$. The only tool available for doing this in the case of QCD is lattice theory, so we must now face directly the issue of extracting renormalon structure of field theories formulated on a (necessarily) finite space-time lattice. 


\section{Finding renormalon singularities on a finite lattice}

If, as we have argued, weak-coupling perturbation theory is not a reliable guide to the full renormalon structure in an infrared unstable theory with dynamical mass generation, it will be necessary to carry out a fully nonperturbative evaluation of the Borel function $B(s)$ for the relevant physical quantity. In QCD, this restricts us to a numerical (Monte Carlo) simulation of the lattice gauge theory. There are two obvious difficulties which must be overcome. Firstly, the information obtained in such a calculation is necessarily subject to statistical errors. Consequently, any technique em-

ployed for the extraction of the singularity structure of $B(s)$ for $\operatorname{Re}(s)>0$ (obtained by analytic continuation from $\operatorname{Re}(s)<0$ ) must be fairly resistant to the inevitably noisy input information. Secondly, simulations can only be performed of systems with a finite number of degrees of freedom, i.e. at finite volume. The Borel Transform $B(s)$ (15) will in this case lack the Type 2 singularities associated with the condensation of zero momentum modes in the theory. In this section we shall explain one possible modification of the definition (15) capable of revealing the precursors of the infinite volume singularity structure on a finite lattice.

As usual, a convenient model for studying the renormalon structure on a finite lattice is the $\mathrm{O}(\mathrm{N})$ sigma model in 2 dimensions: the large $\mathrm{N}$ limit of the theory is analytically solvable, even on the lattice. We shall use this model to gain insight in formulating a modified lattice Borel transform. We 
begin by pointing out a basic difficulty in the definition (15) of the full Borel function $B_{L}(s)$ when the transform is applied to a lattice system with a mass gap generated via logarithmic infrared divergencies. To make the algebra as transparent as possible, consider the toy model of Section 2 where the gap equation is modelled by the discrete sum

$$
\frac{1}{f}=\frac{1}{L} \sum_{n=1}^{L} \frac{1}{m+n / L} \equiv s_{L}(m) \rightarrow \ln \left(\frac{1+m}{m}\right), \quad L \rightarrow \infty
$$

Here $m$ is the analog of the squared dynamical mass and we take $G(m)=$ $\frac{1}{p^{2}+m}$ as our model of the momentum space propagator, with Borel transform

$$
\begin{aligned}
B_{L}(s) & =\int_{0}^{\infty} e^{s / f} G(m(f)) d\left(\frac{1}{f}\right), \quad \operatorname{Re}(s)<0 \\
& =-\int_{0}^{\infty} s_{L}^{\prime}(m) e^{s s_{L}(m)} G(m) d m
\end{aligned}
$$

This integral defines the analytic function $B_{L}(s)$ for $\operatorname{Re}(s)<0$. The problem is that the $L \rightarrow \infty$ limit does not commute with the desired analytic continuation to the right-half $s$-plane. This becomes clear if we subject (59) to an integration by parts

$$
B_{L}(s)=\frac{1}{s} e^{s s_{L}(0)} G(0)+\frac{1}{s} \int e^{s s_{L}(m)} G^{\prime}(m) d m
$$

For $0<\operatorname{Re}(s)<1$, the integral in (60) is now well-defined as $L \rightarrow \infty$ (as the integration is convergent for $m \rightarrow 0$ ), but $s_{L}(0) \sim \ln (L)$ causes a power divergence $\propto L^{s}$ if the large volume limit is taken after the analytic continuation is performed.

In the case of the sigma model at large $N$, where we have analytic control of the theory, one may devise the following cure for this problem, which arises 
from the deviation at finite $L$ in the integration measure for small $m$ from the infinite volume form, Namely, one devises a modified Borel transform on the lattice which has a smooth analytic continuation to $\operatorname{Re}(s)>0$ at large $L$. In the $L \rightarrow \infty$ limit, the integration measure goes over to $m^{-1-s} d m$. Choosing an infrared cutoff $\beta \sim O(1 / L)$ (the precise value is immaterial) we may define an alternative transform of the (nonperturbatively determined!) propagator $G(m)$ on the lattice as

$$
B_{\text {mod }}(s) \equiv e^{\eta s} \int_{0}^{\beta} m^{-1-s} G(m-\beta) d m+B_{L}(s)
$$

with $\eta, \beta$ satisfying $\eta=s_{L}(0)-\ln (1 / \beta)$, i.e

$$
e^{s\left(s_{L}(0)-\eta\right)}=\beta^{-s}
$$

The relation (62) ensures that the integrand $m^{-s} G(m-\beta)$ in the first term in (61) matches (at $m=\beta$ ) smoothly to the corresponding lattice determined integrand $e^{s_{L}(m)} G(m)$ in $B_{L}(s)$. The supplementary contribution generates a term $-\frac{1}{s} e^{\eta s} G(0) \sim L^{s}$ as $L \rightarrow \infty$, exactly cancelling the pathological term in (60). We have checked that the above procedure leads to a numerically stable detection procedure for renormalon singularities using the test case of the 2D sigma model. As input to the Borel transform, the feasibility of the method can be studied by using the analytic large $\mathrm{N}$ results (instead of actual Monte Carlo data). Statistical noise can also be introduced by hand to check the robustness of the procedure. The mass gap equation on a finite lattice for the $2 \mathrm{D}$ sigma model is

$$
\frac{1}{f}=\frac{1}{L^{2}} \sum_{\vec{k}} \frac{1}{d(\vec{k})+m} \equiv \frac{1}{4 \pi} s_{L}(m)
$$


where $\vec{k}=\left(\frac{2 \pi}{L} k_{x}, \frac{2 \pi}{L} k_{y}\right), k_{x}, k_{y}=1,2, . . L$ and $d(\vec{k}) \equiv 4\left(\sin \left(\frac{\pi k_{x}}{L}\right)^{2}+\sin \left(\frac{\pi k_{y}}{L}\right)^{2}\right)$. In the large $\mathrm{N}$ limit the momentum space $\vec{\phi}$ propagator is

$$
G(f ; \vec{q})=\frac{1}{d(\vec{q})+m(f)}
$$

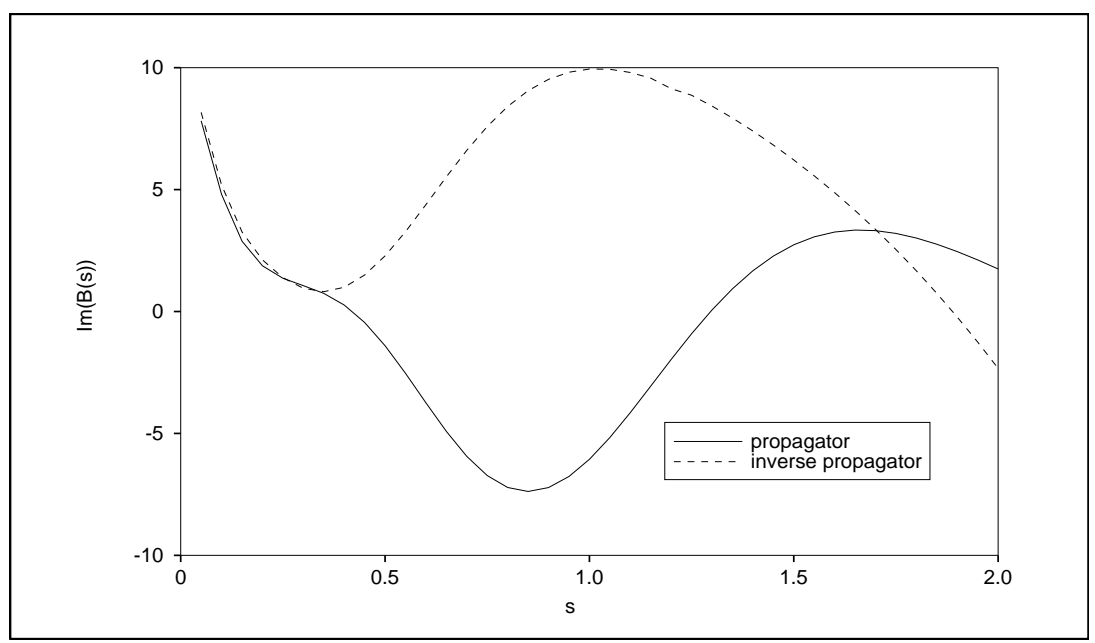

Figure 7: Borel Transform of propagator, inverse propagator on a 30x30 lattice $\left(\gamma=0.1, N_{T}=10\right)$

In analogy to (61), we analytically continue the modified transform (suppressing for notational simplicity the momentum dependence)

$$
B_{\text {mod }}(s) \equiv e^{\eta s} \int_{0}^{\beta} m^{-1-s} G(m(f)) d m-\int_{0}^{c} s_{L}^{\prime}(m) e^{s s_{L}(m)} G(m) d m
$$

with $\beta \sim O\left(1 / L^{2}\right), \eta=s_{L}(0)-\ln (1 / \beta)$. The analytic continuation to positive real $s$ can be performed in a numerically stable way by writing

$$
s=s_{R} e^{w}
$$

and Taylor expanding around $w=i \pi\left(\right.$ where $B_{\bmod }(s)$ is analytic). The circle of convergence has radius $\pi$, as $B_{\text {mod }}$ is analytic in the cut plane, and we 
can evaluate $\operatorname{Im} B_{\text {mod }}\left(s_{R}+i \gamma\right)$ by summing the series to some finite order $N_{T}$ at $w \sim i \gamma$. The result for $N_{T}=10, \gamma=0.1$ on a $30 \times 30$ lattice is shown in Fig(可) for the Borel transform of both $G$ (with $d(\vec{q})=1$ ) and the inverse propagator $G^{-1}$. The effect of keeping more terms in the Taylor expansion is shown in $\operatorname{Fig}(8)$ for the Borel Transform of $G^{-1}$. The peak at the first renormalon becomes sharper as $N_{T}$ increases. However, the results become (for fixed $\gamma$ ) less accurate as $s_{R}$ increases, which requires $w$ to approach the edge of the circle of convergence.

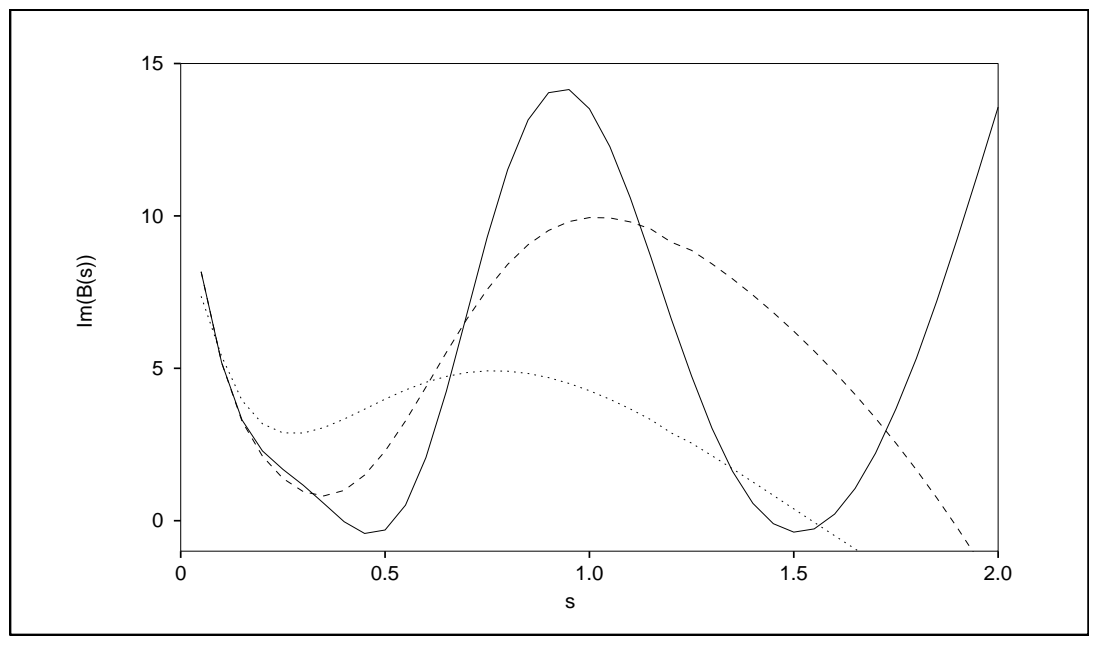

Figure 8: Borel Transform of inverse propagator on a $30 \times 30$ lattice $\left(N_{T}=\right.$ $6,10,14)$

Finally, the sensitivity of the continuation procedure to a random superimposed 1\% statistical noise on $G(m)$ (well within the reach of Monte Carlo simulations, for example) is shown in Fig(9). The first renormalon is still clearly visible at $s \sim 1$, with the effects of the noise becoming serious only for $s>1.5$. In all of these cases, we should emphasize that the first 
term in (65) is absolutely essential to obtain stable results from the analytic continuation.

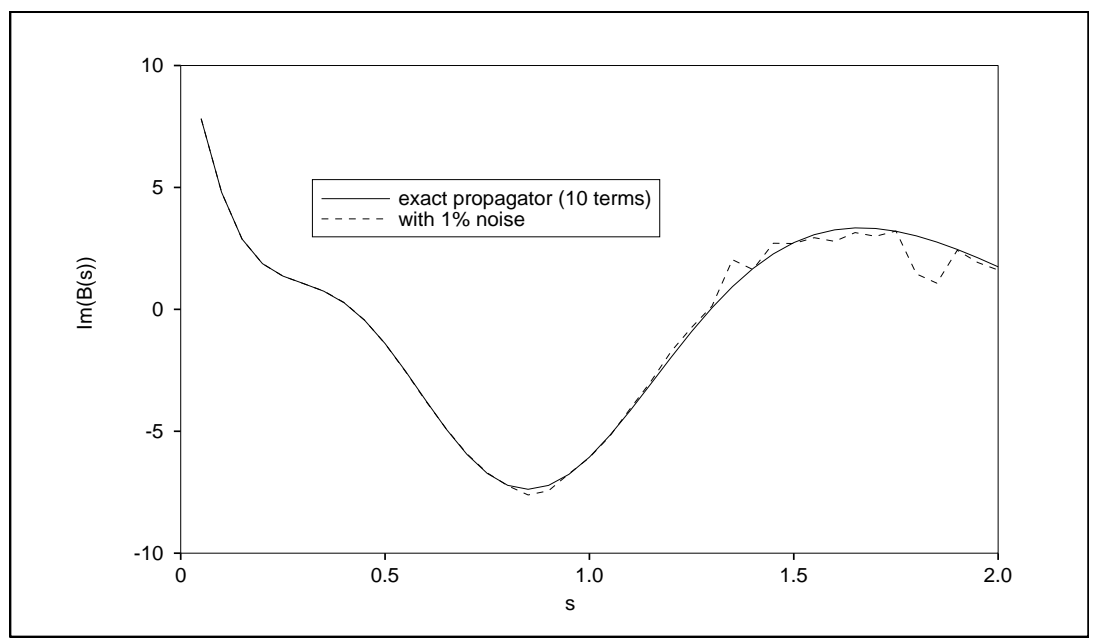

Figure 9: Borel Transform of noisy propagator on a 30x30 lattice $\left(N_{T}=10\right)$

\section{Summary and Discussion}

In this paper we have used the nonlinear sigma model as a convenient analytical platform for examining the interrelationship of various versions of the Borel transform. Infrared unstable theories (like QCD or the sigma model) with dynamical mass generation are inevitably non-Borel summable, and a precise Borel reconstruction of such theories is only possible starting from the generalized Borel transform $B(s)$, defined as the Laplace transform with respect to the inverse bare coupling of the relevant amplitude.(The complex nonperturbative behavior of such theories is entirely connected with the infrared structure, so it suffices to work throughout with an ultraviolet cutoff: 
in the case of QCD, this implies a lattice formulation.)

Unfortunately, the conventional definition of the Borel transform in terms of the formal perturbative series (for which we have used the notation $\hat{B}(s)$ ) does not lead to a precise reconstruction theorem in the nonBorel case. This function extends (in the sense of having identical asymptotic expansion for small $s$ ) to a function $\tilde{B}(s)$, the discontinuity of the generalized transform $B(s)$ introduced above, which does however yield such a reconstruction. The essential difference between the two functions can be seen explicitly in the large $N$ limit of the nonlinear sigma model, where an infrared cutoff in the elementary modes of the theory can be seen to eliminate the conventional infrared renormalons of perturbation theory, while leaving the singularities in $B(s)$ intact.

The necessity for a fully nonperturbative approach to the generalized Borel function $B(s)$ leads us to the consideration of simulations on a finite lattice, which is often the only tool available for reliable nonperturbative calculation. The Borel singularities on the positive $s$ axis have been seen to fall into two types. Type 1 singularities are present (as actual singularities) already in the finite volume theory: however they are numerically camouflaged by perturbative prefactors in disconnected Green's functions. Finding such singularities on a finite lattice will therefore require removing disconnected vacuum contributions (cf. Section 4). Type 2 singularities (the usual IR renormalons fall into this category) are strictly speaking only present in the infinite volume limit. Moreover, the interchange of analytic continuation to $\operatorname{Re}(s)>0$ and the infinite volume limit is not uniform, requiring a modification (discussed in Section 6) of the definition of the Borel Transform 
on a finite lattice in order to allow detection of the renormalon precursors. Such a modification indeed allows us to extract the location of renormalon singularities for the analytically tractable test case of the sigma model at large $N$. The application to situations (say, the sigma model with $N=3$, or quenched QCD) where Monte Carlo methods must be employed to compute the nonperturbative structure of the theory is presently under study. 


\section{Acknowledgements}

A.D. gratefully acknowledges many useful and enlightening conversations on the role of renormalons with A.H. Mueller.

\section{References}

[1] F.J. Dyson, Phys. Rev. 85, 631(1952).

[2] C.M. Bender and T.T. Wu, Phys. Rev. 184,1231(1969).

[3] Eckmann, J.-P., J. Magnen and Seneor, R., Comm. Math. Phys. 39, 251(1975); J. Magnen and R. Seneor, Comm. Math. Phys. 56, 237(1977).

[4] A. Duncan and Jones, H.F., Phys. Rev. D47, 2560(1993).

[5] G. 't Hooft, Erice Lectures 1977, A. Zichichi ed. (Plenum, New York 1979).

[6] A.H. Mueller, Phys. Lett. B308, 355(1993); Nucl. Phys. B250, 327(1985).

[7] A useful reprint volume is Large Order Behaviour of Perturbation Theory, J.C. Le Guillou and J. Zinn-Justin eds.(North Holland, Amsterdam, 1989). 
[8] "Ultraviolet Renormalon Calculus", A.I. Vainshtein and V.I. Zakharov, TPI-MINN-94-9-T, Minnesota preprint, Apr. 1994.

[9] W.Y. Crutchfield, Phys. Rev. D19, 2370(1979).

[10] F. David, Nucl. Phys. B209, 433(1982).

[11] L.S. Brown, Yaffe, L.G., and C. Zhai, Phys. Rev. D46, 4712(1992).

[12] M. Beneke and V.I. Zakharov, Phys.Lett. B312, 340(1993); M. Beneke, Phys.Lett. B307, 154(1993); M. Beneke, Nucl. Phys. B405, 424(1993).

[13] J. Zinn-Justin, in Recent Advances in Field Theory and Statistical Mechanics, Proceedings of the Les Houches Summer School, Les Houches, France, 1982, edited by J.B. Zuber and R. Stora (North Holland, Amsterdam,1983), p.40.

[14] See Aspects of Symmetry, Selected Erice Lectures of S. Coleman, (Cambridge University Press, 1985), p.351. 